\title{
A Renormalisation Group Approach to the Universality of Wigner's Semicircle Law for Random Matrices with Dependent Entries
}

\author{
Thomas Krajewski \\ Centre de Physique Théorique, Aix-Marseille Université, Marseille, France \\ Correspondence should be addressed to Thomas Krajewski; krajewsk@gmail.com
}

Received 15 June 2017; Revised 1 September 2017; Accepted 1 October 2017; Published 31 December 2017

Academic Editor: Ralf Hofmann

Copyright (C) 2017 Thomas Krajewski. This is an open access article distributed under the Creative Commons Attribution License, which permits unrestricted use, distribution, and reproduction in any medium, provided the original work is properly cited. The publication of this article was funded by SCOAP $^{3}$

We show that if the non-Gaussian part of the cumulants of a random matrix model obeys some scaling bounds in the size of the matrix, then Wigner's semicircle law holds. This result is derived using the replica technique and an analogue of the renormalisation group equation for the replica effective action.

\section{Introduction}

Random matrix theory (see the classical text [1]) first appeared in physics in Wigner's work on the level spacing in large nuclei. Since then, it has proven to have multiple applications to physics and other branches of science (see, e.g., [2]). Most of these applications rely on the universal behaviour of some of the observables for matrices of large size. A simple example is Wigner's semicircle law for the eigenvalue density that holds in the large $N$ limit for matrices whose entries are independent and identically distributed.

Understanding the universal behaviour of eigenvalue distributions and correlations ranks among the major problems in random matrix theory. In this respect, the renormalisation group turns out to be a powerful technique. Introduced in the context of critical phenomena in statistical mechanics by $\mathrm{K}$. Wilson to account for the universality of critical exponents, the latter has also been proven to be useful in understanding probability theory. For instance, it leads to an insightful proof of the central limit theorem (see the review by Jona-Lasinio [3] and references therein).

The renormalisation group has been used to derive the semicircle law for random matrices in the pioneering work of Brézin and Zee [4]. In the latter approach, the renormalisation group transformation consists in integrating over the last line and column of a matrix of size $N+1$ to reduce it to a matrix of size $N$. This leads to a differential equation for the resolvent $G(z)=1 / N\left\langle\operatorname{Tr}(z-M)^{-1}\right\rangle$ in the large $N$ limit whose solution yields the semicircle law.

In this paper, we follow a different route: we first express the resolvent as an integral over replicas and introduce a differential equation for the replica effective action. This differential equation is a very simple analogue of Polchinski's exact renormalisation group equation [5]. It is used to derive inductive bounds on the various terms, ensuring that the semicircle law is obeyed provided the cumulants of the original matrix model fulfil some simple scaling bounds in the large $N$ limit.

This paper is based on some work in collaboration with Krajewski et al. in which we extend Wigner's law to random matrices whose entries fail to be independent [6] to which we refer for further details. There have been other works on such an extension (see [7-9]).

\section{What Are Random Matrices?}

A random matrix is a probability law on a space of matrices, usually given by the joint probability density on its entries:

$$
\rho(M)=\rho\left(M_{11}, M_{12}, \ldots\right) .
$$


Thus, a random matrix of size $N$ is defined as a collection of $N^{2}$ random variables. However, there is a much richer structure than this, relying notably on the spectral properties of the matrices.

Here, we restrict our attention to a single random matrix. Note that it is also possible to consider several random matrices, in which case the noncommutative nature of matrix multiplication plays a fundamental role, leading to the theory of noncommutative probabilities.

There are two important classes of probability laws on matrices:

(i) Wigner ensemble: the entries are all independent variables:

$$
\rho(M)=\prod_{i, j} \rho_{i j}\left(M_{i j}\right),
$$

up to the Hermitian condition $\bar{M}_{i j}=M_{j i}$.

(ii) Unitary ensemble: the probability law is invariant under unitary transformations:

$$
\rho\left(U M U^{\dagger}\right)=\rho(M),
$$

for any unitary matrix $U \in U(N)$.

The only probability laws that belong to both classes are the Gaussian ones:

$$
\rho(M) \propto \exp -\frac{1}{2 \sigma^{2}} \operatorname{Tr}(M)^{2},
$$

up to a shift of $M$ by a fixed scalar matrix.

The main objects of interest are the expectation values of observables, defined as

$$
\langle\mathcal{O}\rangle=\int d M \rho(M) \mathcal{O}(M)
$$

Among the observables, the spectral observables defined as symmetric functions of the eigenvalues of $M$ play a crucial role in many applications. This is essentially due to their universal behaviour: in the large $N$ limit, for some matrix ensembles and in particular regimes, the expectation values of specific spectral observables do not depend on the details of the probability law $\rho(M)$.

Universality is at the root of the numerous applications to physics and other sciences, since the results we obtain are largely model-independent. Among the applications to physics, let us quote the statistics of energy levels in heavy nuclei, disordered mesoscopic systems, quantum chaos, chiral Dirac operators, and so forth.

\section{Wigner's Semicircle Law}

In this paper, we focus on the eigenvalue density, defined as

$$
\rho(\lambda)=\frac{1}{N}\left\langle\sum_{1 \leq i \leq N} \delta\left[\lambda-\lambda_{i}\left(\frac{M}{\sqrt{N}}\right)\right]\right\rangle .
$$

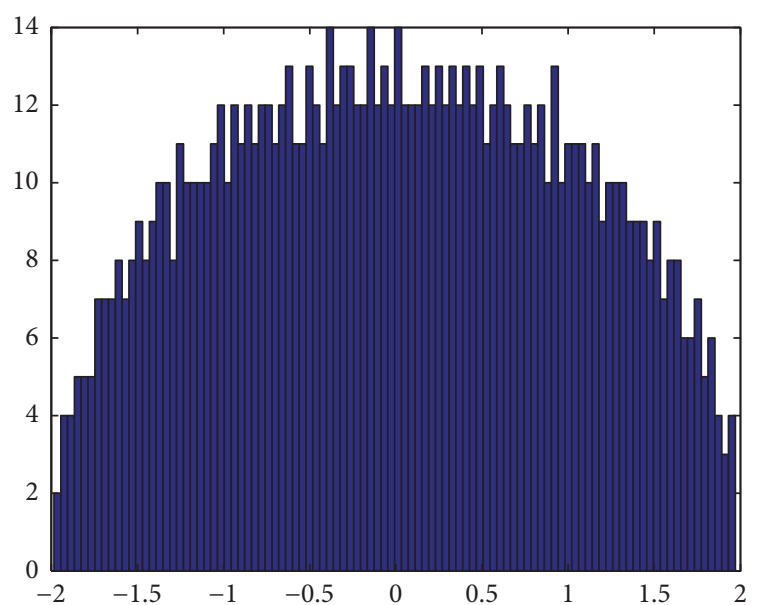

Figure 1: Spectrum of a large $(N=1000)$ Hermitian matrix with a Gaussian distribution $(\sigma=1)$.

In particular, a universal behaviour is expected in the large $N$ limit for some ensembles.

For a Gaussian random Hermitian matrix $\rho(M) \propto$ $\exp -\left(1 / 2 \sigma^{2}\right) \operatorname{Tr}\left(M^{2}\right)$, the eigenvalue density obeys Wigner's semicircle law:

$$
\begin{aligned}
\lim _{N \rightarrow \infty} \int_{\mathbb{R}} d \lambda \lambda^{k} \rho(\lambda) \\
\quad= \begin{cases}\frac{1}{2 \pi \sigma^{k+2}} \int_{-2 \sigma}^{2 \sigma} d \lambda \lambda^{k} \sqrt{4 \sigma^{2}-\lambda^{2}}, & \text { if } k \text { is even, } \\
0, & \text { if } k \text { is odd. }\end{cases}
\end{aligned}
$$

Empirically, $\rho(\lambda)$ may be determined by plotting the histogram of eigenvalue of a matrix taken at random with a given probability law (see Figure 1).

The derivation of Wigner's semicircle in the large $N$ limit is based on the resolvent (also known as the Green function):

$$
\begin{aligned}
G(z) & =\lim _{N \rightarrow+\infty} \frac{1}{N}\left\langle\operatorname{Tr}\left(z-\frac{M}{\sqrt{N}}\right)^{-1}\right\rangle \\
& =\frac{z}{2 \sigma^{2}}\left(1-\sqrt{1-\frac{4 \sigma^{2}}{z^{2}}}\right) .
\end{aligned}
$$

Then, the density of eigenvalues is recovered as

$$
\rho(\lambda)=\frac{G\left(\lambda-\mathrm{i} 0^{+}\right)-G\left(\lambda+\mathrm{i} 0^{+}\right)}{2 \mathrm{i} \pi},
$$

where we have used the relation

$$
\frac{1}{x \pm \mathrm{i} 0^{+}}=\text {p.v. } \frac{1}{x} \mp 2 \mathrm{i} \pi \delta(x) .
$$

In the large $N$ limit, for the Gaussian model, the resolvent obeys the self-consistency equation (also known as the Schwinger-Dyson equation) (see, e.g., [10], Section VII.4):

$$
G(z)=\sum_{k=0}^{\infty} \frac{\sigma^{k} G^{k}(z)}{z^{k+1}}=\frac{1}{z-\sigma G(z)} .
$$


Its solution that behaves as $1 / z$ for large $z$ is

$$
G(z)=\frac{z}{2 \sigma^{2}}\left(1-\sqrt{1-\frac{4 \sigma^{2}}{z^{2}}}\right) .
$$

Taking the cut of the square root on the negative real axis, we obtain Wigner's semicircle law (7) in the large $N$ limit.

The semicircle law is not limited to the Gaussian case; it also holds for Wigner matrices in the large $N$ limit. A random Hermitian $N \times N$ matrix is a Wigner matrix if

(i) real and imaginary parts of upper diagonal elements are independent and identically distributed (i.i.d.) with mean 0 and variance $\sigma$;

(ii) diagonal elements are i.i.d. with finite mean and variance and independent of the off-diagonal ones.

Then, in the limit $N \rightarrow+\infty$, the eigenvalue distribution of $M / \sqrt{N}$ is the semicircle law (7).

The original proof is of combinatorial nature and involves the expectation of the moments:

$$
\begin{aligned}
& \lim _{N \rightarrow+\infty} \frac{1}{N^{k / 2+1}}\left\langle\operatorname{Tr}\left(M^{k}\right)\right\rangle \\
& \quad= \begin{cases}\frac{(2 l) !}{(l !)^{2}(l+1)}, & \text { for } k=2 l \text { even, } \\
0, & \text { for } k \text { odd. }\end{cases}
\end{aligned}
$$

To derive this result, the idea is to first factorise $\rho$ for a Wigner ensemble as

$$
\rho(M)=\prod_{i} \rho^{\prime}\left(M_{i i}\right) \prod_{i<j} \rho^{\prime \prime}\left(\operatorname{Re} M_{i j}\right) \rho^{\prime \prime}\left(\operatorname{Im} M_{i j}\right),
$$

where $\rho^{\prime}$ is the common probability density of the real diagonal terms and $\rho^{\prime \prime}$ is the common probability density of the real and imaginary parts of the off-diagonal terms.

Then, we expand the trace and integrate over the independent real variables $M_{i i}$, $\operatorname{Re} M_{i j}$, and $\operatorname{Im} M_{i j}$. The power of $N$ in the expectation of a given moment arises from the denominator $1 / N^{k / 2+1}$ and from the number of independent indices in the summations. In the large $N$ limit, the only configurations that survive are counted by Catalan numbers: $C_{l}=(2 l) ! /(l !)^{2}(l+1)$. Since the latter also appear in the Taylor expansion,

$$
\frac{z}{2 \sigma^{2}}\left(1-\sqrt{1-\frac{4 \sigma^{2}}{z^{2}}}\right)=\sum_{l \geq 0} \frac{(2 l) !}{(l !)^{2}(l+1)} \frac{\sigma^{2 l}}{z^{2 l+1}},
$$

we conclude that

$$
\begin{aligned}
G(z) & =\lim _{N \rightarrow+\infty} \frac{1}{N}\left\langle\operatorname{Tr}\left(z-\frac{M}{\sqrt{N}}\right)^{-1}\right\rangle \\
& =\lim _{N \rightarrow+\infty} \sum_{k=0}^{\infty} \frac{1}{z^{k+1}} \frac{1}{N^{k / 2+1}}\left\langle\operatorname{Tr}\left(M^{k}\right)\right\rangle \\
& =\frac{z}{2 \sigma^{2}}\left(1-\sqrt{1-\frac{4 \sigma^{2}}{z^{2}}}\right) .
\end{aligned}
$$

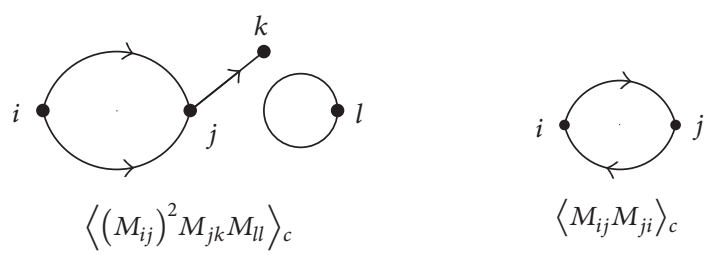

FIGURE 2: Examples of graph associated with cumulants.

This is the form of the resolvent which leads to Wigner's semicircle law. Here, we see universality at work: in the large $N$ limit, the eigenvalue density is given by the semicircle law, whatever the probability densities $\rho^{\prime}$ and $\rho^{\prime \prime}$ are. However, this result relies on the independence of the matrix elements. In the next section, we will extend it to matrices whose entries are not necessary independent.

\section{Wigner's Law beyond Wigner Ensembles}

Let us introduce the cumulants, defined through their generating function:

$$
\begin{aligned}
& \left\langle M_{i_{1} j_{1}} \cdots M_{i_{k} j_{k}}\right\rangle_{c} \\
& \quad=\left.\frac{\partial}{\partial J_{j_{1} i_{1}}} \cdots \frac{\partial}{\partial J_{j_{k} i_{k}}} \log \langle\exp \operatorname{Tr}(M J)\rangle\right|_{J=0} .
\end{aligned}
$$

In the physics terminology, these are the connected correlation functions. In particular, the Gaussian cumulants vanish beyond the quadratic term:

$$
\begin{aligned}
\rho(M) & \propto \exp -\frac{1}{2 \sigma^{2}} \operatorname{Tr}\left(M^{2}\right) \\
& \Longrightarrow\left\{\begin{array}{l}
\left\langle M_{i j} M_{k l}\right\rangle_{c}=\sigma^{2} \delta_{i l} \delta_{j k} \\
\text { vanish otherwise. }
\end{array}\right.
\end{aligned}
$$

Therefore, cumulants of degree higher than 2 are a measure of the deviation from the Gaussian case.

Turning back to the general case, for each cumulant, we construct an oriented graph as follows (see Figure 2 for some examples):

(i) Vertices are distinct matrix indices in the cumulant.

(ii) There is an edge from $i$ to $j$ for every $M_{i j}$.

Since nonquadratic cumulants measure deviations from the Gaussian case, if the perturbation is small, it is reasonable to expect that the semicircle law is still obeyed.

To state this result, recall that an oriented graph is Eulerian if every vertex has an equal number of incoming and outgoing edges. Equivalently, it means that every connected component admits an Eulerian cycle, that is, an oriented cycle that passes through all edges, respecting the orientation. Furthermore, let us denote by $v(G), e(G)$, and $c(G)$ the numbers of vertices, edges, and connected components of $G$, respectively. 
Theorem 1 (Wigner's law for matrices with dependent entries). Let $\rho_{N}$ be a probability law on the space of Hermitian $N \times N$ matrices $M$ such that its cumulants can be decomposed as $C_{G}=C_{G}^{\prime}+C_{G}^{\prime \prime}$, with $C_{G}^{\prime}$ being a Gaussian cumulant and $C_{G}^{\prime \prime}$ being a perturbation such that, uniformly in the vertex indices $i_{1}, \ldots, i_{v(G)}$ (i.e., all constants involved should not depend on these indices),

(i) $\lim _{N \rightarrow \infty} N^{v(G)-c(G)-e(G) / 2} C_{G}^{\prime \prime}\left(i_{1}, \ldots, i_{v(G)}\right)=0$ if $G$ is Eulerian,

(ii) $N^{v(G)-c(G)-e(G) / 2} C_{G}^{\prime \prime}\left(i_{1}, \ldots, i_{v(G)}\right)$ is bounded if $G$ is not Eulerian.

Then, the moments of the eigenvalue distribution of the matrix $M / \sqrt{N}$ converge towards the moments of the semicircle law, with $\sigma$ given by the Gaussian cumulant $\left\langle M_{i j} M_{k l}\right\rangle_{c}=\sigma^{2} \delta_{i l} \delta_{j k}$ :

$$
\begin{aligned}
& \lim _{N \rightarrow \infty} \int_{\mathbb{R}} d \lambda \lambda^{k} \rho_{N}(\lambda) \\
& \quad= \begin{cases}\frac{1}{2 \pi \sigma^{k+2}} \int_{-2 \sigma}^{2 \sigma} d \lambda \lambda^{k} \sqrt{4 \sigma^{2}-\lambda^{2}} & \text { if } k \text { is even, } \\
0 & \text { if } k \text { is odd. }\end{cases}
\end{aligned}
$$

For instance, for the graph<smiles>[X][Te]1([Al]2CCCC2)CCCC1</smiles>

which is not Eulerian, with $v=3, e=4$, and $c=2$, the cumulant should obey

$$
\frac{1}{N}\left|\left\langle\left(M_{i j}\right)^{2} M_{j k} M_{l l}\right\rangle_{c}\right| \leq K
$$

with $K$ being a constant that does not depend on the indices $i, j, k$, and $l$. On the other hand, for the graph

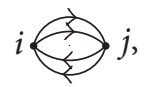

which is Eulerian, with $v=2, e=4$, and $c=1$, we impose

$$
\lim _{N \rightarrow+\infty} \frac{1}{N}\left|\left\langle\left(M_{i j}\right)^{2}\left(M_{j i}\right)^{2}\right\rangle_{c}\right|=0
$$

uniformly in $i$ and $j$.

As an illustration, we recover the case of Wigner matrices (with finite moments). Indeed,

(i) there is no graph with $v \geq 3$ (independence of off diagonal matrix elements);

(ii) for $v=1$ and $v=2, e \geq 3$, bounds are satisfied because of $1 / N^{e / 2}$ and all moments are assumed to be finite;

(iii) $C_{i \bullet \bullet} \cdot j(i, j)=\left\langle M_{i j}\right\rangle_{c}=\left\langle M_{i j}\right\rangle=0$ (off diagonal elements have mean value 0 );

(iv) ${ }^{C}{ }_{i} \bigcirc j \bigcirc(i, j)=\left\langle M_{i i} M_{j j}\right\rangle_{c}=\left\langle M_{i i} M_{j j}\right\rangle-\left\langle M_{i i}\right\rangle\left\langle M_{j j}\right\rangle=0$ (independence of diagonal elements);

(v) $C_{i \bullet} \mapsto j(i, j)=\left\langle M_{i j} M_{j j}\right\rangle_{c}=\left\langle M_{i j} M_{j j}\right\rangle-\left\langle M_{i j}\right\rangle\left\langle M_{j j}\right\rangle=0$ (independence of diagonal and off diagonal elements);

(vi)

$C_{i}{ }^{j}(i, j)=\left\langle M_{i j} M_{i j}\right\rangle_{c}=\left\langle M_{i j} M_{i j}\right\rangle-\left\langle M_{i j}\right\rangle\left\langle M_{i j}\right\rangle=\left\langle\left(\operatorname{Re} M_{i j}\right)^{2}-\left(\operatorname{Im} M_{i j}\right)^{2}\right\rangle+2 \mathrm{i}\left\langle\operatorname{Re} M_{i j} \operatorname{Im} M_{i j}\right\rangle=0$ (independence of real and imaginary parts and equality of their distributions with mean value 0$)$;

$$
{ }^{C}=\sigma^{2} \text { is the Gaussian cumulant leading to the semicircle law. }
$$

The case of unitarily invariant matrices is critical since the bounds are saturated (see [6]). This is consistent since we know that the semicircle law is not obeyed by unitary nonGaussian ensembles [11].

It is possible to give a combinatorial proof of this result based on the relation between moments and cumulants:

$$
\begin{aligned}
& \left\langle M_{i_{1} j_{1}} \cdots M_{i_{k} j_{k}}\right\rangle \\
& =\sum_{\substack{I_{1}, \ldots, I_{p} \\
\left\{\left(i_{1}, j_{1}\right), \ldots,\left(i_{k}, j_{k}\right)\right\}}}\left\langle\prod_{i j \in I_{1}} M_{i j}\right\rangle_{c} \cdots\left\langle\prod_{i j \in I_{p}} M_{i j}\right\rangle_{c} .
\end{aligned}
$$

In the moment method, we have to estimate

$$
\begin{aligned}
& \frac{1}{N^{k / 2+1}}\left\langle\operatorname{Tr}\left(M^{k}\right)\right\rangle \\
& \quad=\frac{1}{N^{k / 2+1}} \sum_{1 \leq i_{1}, \ldots, i_{k} \leq N}\left\langle M_{i_{1} i_{2}} \cdots M_{i_{k} i_{1}}\right\rangle .
\end{aligned}
$$

Then, we express the moments in (23) in terms of cumulants using (22) and represent each cumulant as a graph. Because of the trace, one has to draw Eulerian cycles on the graphs after some vertex identifications. Then, the scaling bounds on the cumulants can be used to show that only Gaussian terms survive. 


\section{Proof Based on the Replica Effective Action}

Let us give a renormalisation group proof of this result based on the replica effective action. The use of replicas in random matrix theory is a classical subject (see, e.g., [12] or [13]). To begin with, let us note that

$$
\operatorname{Tr}\left(z-\frac{M}{\sqrt{N}}\right)^{-1}=\frac{\partial}{\partial z} \log \operatorname{det}\left(z-\frac{M}{\sqrt{N}}\right) .
$$

It is convenient to express the logarithm using the replica method. First, observe that

$$
\log (A)=\lim _{n \rightarrow 0} \frac{A^{n}-1}{n} .
$$

Then, we express the $n$th power of the determinant as a Gaussian integral over $n$ replicas of a complex vector of size $N$ (with a factor of $\pi^{n N}$ included in the measure):

$$
\frac{1}{\operatorname{det}^{n}(z-M)}=\int d X \exp -\operatorname{Tr}\left(X^{\dagger}(z-M) X\right),
$$

which fit into an $N \times n$ complex matrix $X=\left(X_{i, a}\right)_{\substack{1 \leq i \leq N \\ 1 \leq a \leq n}}$.

The limit $n \rightarrow 0$ may be worrisome; its meaning is as follows. Because of $U(n)$ invariance, any perturbative result in powers of $1 / z$ is a polynomial in $n$, from which we retain only the linear term. Of course, this may not hold beyond perturbation theory, where replica symmetry breaking can occur.

After averaging over $M$ with the random matrix density $\rho(M)$, we obtain the following expression for the resolvent:

$$
\begin{aligned}
& G(z) \\
& =-\frac{1}{N} \frac{\partial}{\partial z}\left[\int d X \exp \left\{-\operatorname{Tr}\left(X^{\dagger} X\right)+V_{0}(X)\right\}\right]_{\substack{\text { order } 1 \\
\text { in } n}},
\end{aligned}
$$

where the replica potential is

$$
V_{0}(X)=\log \left\langle\exp \operatorname{Tr}\left(X^{\dagger} \frac{M}{\sqrt{N}} X\right)\right\rangle .
$$

Because of the logarithm, the potential involves the cumulants and can be expanded over graphs as

$$
\begin{aligned}
V_{0}(X)= & \sum_{\substack{G \\
\text { oriented graph }}} \frac{1}{|\operatorname{Aut}(G)| N^{e(G) / 2}} \\
& \cdot \sum_{\substack{1 \leq i_{1}, \ldots, i_{v(G)} \leq N \\
\text { all different }}} C_{G}\left(i_{1}, \ldots, i_{v(G)}\right) \\
& \cdot \prod_{e \text { edge }}\left(X X^{\dagger}\right)_{i_{s(e)} i_{t(e)}},
\end{aligned}
$$

where $s(e)$ is the source of edge $e$ and $t(e)$ is its target.

Let us introduce a replica effective action, obtained by a partial integration:

$$
V(t, X)=\log \int d Y \exp \left\{-\frac{\operatorname{Tr}\left(Y^{\dagger} Y\right)}{t}+V_{0}(X+Y)\right\}
$$

$-N n \log t$

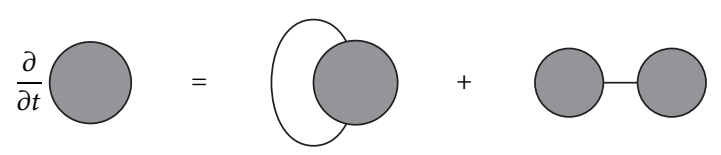

FIGURE 3: Graphical interpretation of the renormalisation group equation.

The parameter $t$ ranges between 0 (where we have no integration, $\left.V(t=0, X)=V_{0}(X)\right)$ and $t=1 / z$.

The effective potential obeys a semigroup property that follows from Gaussian convolution (see, e.g., [14], Section A10.1):

$$
\begin{aligned}
& V(t+s, X) \\
& =\log \int d Y \exp \left\{-\frac{\operatorname{Tr}\left(Y^{\dagger} Y\right)}{s}+V(t, X+Y)\right\} \\
& \quad-N n \log s .
\end{aligned}
$$

For small $s=d t$, it translates into the following renormalisation group equation, which is a simple version of Polchinski's exact renormalisation group equation [5]:

$$
\frac{\partial V(t, X)}{\partial t}=\sum_{i, a}\left(\frac{\partial^{2} V(t, X)}{\partial X_{i, a} \partial \bar{X}_{i, a}}+\frac{\partial V(t, X)}{\partial X_{i, a}} \frac{\partial V(t, X)}{\partial \bar{X}_{i, a}}\right)
$$

The first term on the RHS is referred to as the loop term, since it creates a new loop in the Feynman graph expansion of the effective action, while the second inserts a one-particle reducible line and is referred to as the tree term (see Figure 3).

Taking into account the boundary condition $V(t=0$, $X)=V_{0}(X)$, it is convenient to write (32) in integral form:

$$
\begin{aligned}
& V(t, X) \\
& =V_{0}(X) \\
& \quad+\int_{0}^{t} d s \sum_{i, a}\left(\frac{\partial^{2} V(s, X)}{\partial X_{i, a} \partial \bar{X}_{i, a}}+\frac{\partial V(s, X)}{\partial X_{i, a}} \frac{\partial V(s, X)}{\partial \bar{X}_{i, a}}\right) .
\end{aligned}
$$

This allows us to derive inductive bounds in powers of $t=$ $1 / z$.

From a physical point of view, we evaluate the effective potential by a large succession of small partial integrations, with a total weight given by $t$. Let us stress that, in our context, this differential equation is merely a tool to control the $t$ dependence of the effective action after integrating with a $t$ dependent propagator. 


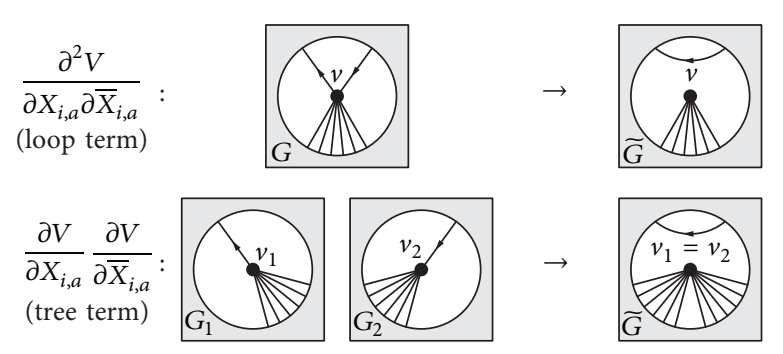

FIGURE 4: Action of the differential operators on the vertices of the effective action.

The effective potential also admits an expansion over graphs:

$$
\begin{aligned}
V(t, X)= & \sum_{\substack{G \\
\text { oriented graph }}} \frac{1}{|\operatorname{Aut}(G)| N^{e(G) / 2}} \\
& \cdot \sum_{\substack{1 \leq i_{1}, \ldots, i_{v(G)} \leq N \\
\text { all different }}} C_{G}\left(t ; i_{1}, \ldots, i_{v(G)}\right) \\
& \cdot \prod_{e \text { edge }}\left(X X^{\dagger}\right)_{i_{s(e)} i_{t(e)}} \cdot
\end{aligned}
$$

This leads to a graphical interpretation of the action of the two differential operators in the renormalisation group equation (see Figure 4). Indeed, in the expansion (see (34)), an edge joining a vertex carrying label $i$ to a vertex carrying $j$ is equipped with a factor $\sum_{a} X_{i, a} \bar{X}_{j, a}$, with $a$ being a replica index. Then, the differential operator $\partial / \partial X_{i, a}$ (resp., $\partial / \partial \bar{X}_{j, a}$ ) removes the outgoing (resp., incoming) half edge. Finally, the remaining half edges are reattached and the vertices identified to yield a new graph on the RHS of (33), with one less edge. These operations are performed on the same graph for the loop term and on distinct ones for the tree term.

Let us decompose the effective cumulants appearing in (34) into Gaussian ones and perturbations and expand both in a power series in $t=1 / z$ :

$$
C_{G}(t)=\sum_{k=0}^{\infty} t^{k}[\underbrace{C_{G}^{\prime(k)}}_{\text {Gaussian }}+\underbrace{C_{G}^{\prime \prime(k)}}_{\text {perturbation }}] \text {. }
$$

The Gaussian terms are those that are constructed using only the Gaussian term in the initial potential $V_{0}(X)$. Even if $V_{0}(X)$ is quartic in $X$, this does not hold for the Gaussian part of $V_{t}(X)$, which contains terms of all orders. The perturbation collects all the remaining terms; they contain at least one nonGaussian perturbation from $V_{0}(X)$.

The renormalisation group equation (33) allows us to prove inductively on $k$ that the perturbations $C_{G}^{\prime \prime(k)}$ obey the same scaling bound imposed on $C_{G}^{\prime \prime(0)}=C_{G}^{\prime \prime}(0)$ and that the purely Gaussian terms do not grow too fast. (i) $\lim _{N \rightarrow \infty} N^{v(G)-c(G)-e(G) / 2}\left[C_{G}^{\prime \prime(k)}\right]_{\text {in } n}^{\operatorname{order} 0}=0$ if $G$ is
Eulerian. (ii) $N^{v(G)-c(G)-e(G) / 2}\left[C_{G}^{\prime \prime(k)}\right]_{\substack{\operatorname{order} 0 \\ \text { in } 0}}$ is bounded if $G$ is not Eulerian.

(iii) $N^{v(G)-c(G)-e(G) / 2}\left[C_{G}^{\prime(k)}\right]_{\substack{\operatorname{order} 0 \\ \text { on } 0}}$ is bounded for any $G$.

This involves a combinatorial discussion based on the graphical interpretation of Figure 4 which can be found in [6]. Let us simply mention that the terms that may violate the bounds are of higher order in $n$. Thus, they are harmless when taking the limit $n \rightarrow 0$ before the limit $N \rightarrow+\infty$.

Finally, using (27) and the renormalisation group equation (33), the resolvent can be expressed as

$$
G(z)=\frac{1}{z}+\frac{1}{N^{3 / 2} z^{2}} \sum_{1 \leq i \leq N}\left[C_{i \curvearrowleft} \bigcirc\left(\frac{1}{z} ; i\right)\right]_{\operatorname{order} 0 \text { in } n}
$$

The scaling bounds for the non-Gaussian cumulants impose, perturbatively in $1 / z$,

$$
\lim _{N \rightarrow \infty} \frac{1}{\sqrt{N}}\left[C_{i \bigodot}^{\prime \prime}\left(\frac{1}{z} ; i\right)\right]_{\text {order } 0 \text { in } n}=0
$$

Therefore, only the Gaussian cumulants contribute and we recover Wigner's semicircle law.

\section{Conclusion and Outlook}

In this paper, we have argued that Wigner's semicircle law remains valid for matrices with dependent entries. The deviation from the independent case is measured by the joint cumulants of the entries, which are assumed to fulfil some scaling bound for large $N$. To establish this result, we have introduced an effective action for the replicas. This effective action obeys a renormalisation group equation that allowed us to prove perturbative bounds on the effective cumulants. As a consequence of these bounds, only the Gaussian terms contribute in the large $N$ limit, thus establishing the validity of Wigner's semicircle law.

It may also be of interest to investigate the case of the sum of a random matrix $M$ and a deterministic one $A$ (see, e.g., [13], where such a model is discussed). In this case, the resolvent is expressed as 


$$
G(z)=-\frac{1}{N} \frac{\partial}{\partial z}\left[\int d X \exp \left\{-\operatorname{Tr}\left(X^{\dagger}(A+z) X\right)+V_{0}(X)\right\}\right] \underset{\text { in } n}{\operatorname{order} 1}
$$

In our context, the deterministic matrix $A$ induces a nontrivial kinetic for the replicas. In particular, if $A$ is a discrete Laplacian, it yields a nontrivial renormalisation group flow that bears some similarities with the QFT renormalisation group. In this case, we expect to exploit the true power of the renormalisation group equation, with a discussion of fixed points and scaling dimensions.

\section{Disclosure}

This paper was presented at the 5th Winter Workshop on Non-Perturbative Quantum Field Theory, Sophia Antipolis, March 2017.

\section{Conflicts of Interest}

The author declares that there are no conflicts of interest regarding the publication of this paper.

\section{Acknowledgments}

The author wishes to thank the organisers of the workshop, Mario Gattobigio, Thierry Grandou, and Ralf Hoffmann, for their kind invitation, as well as Stan Brodsky for insightful remarks. The author is also grateful to his collaborators, Dinh Long Vu and Adrian Tanasa.

\section{References}

[1] M. L. Mehta, Random Matrices, Academic Press, 2004.

[2] G. Akemann, J. Baik, and Ph. Di Francesco, Eds., Oxford Handbook of Random Matrix Theory, Oxford Univ. Press, 2011.

[3] G. Jona-Lasinio, "Renormalisation group and probability theory Phys," Physics Reports, vol. 352, article 0009219, 439 pages, 2001, https://arxiv.org/abs/cond-mat/0009219.

[4] E. Brézin and A. Zee, "Universality of the correlations between eigenvalues of large random matrices," Nuclear Physics B, vol. 402, no. 3, pp. 613-627, 1993.

[5] J. Polchinski, "Renormalization and effective lagrangians," Nuclear Physics B, vol. 231, no. 2, pp. 269-295, 1984.

[6] T. Krajewski, A. Tanasa, and D. L. Vu, "Wigner law for matrices with dependent entries: A perturbative approach," Journal of Physics A: Mathematical and Theoretical, vol. 50, no. 16, 2017, https://arxiv.org/abs/1609.01873.

[7] J. H. Schenker and H. Schulz-Baldes, "Semicircle law and freeness for random matrices with symmetries or correlations," Mathematical Research Letters, vol. 12, no. 4, pp. 531-542, 2005.

[8] K. Hofmann-Credner and M. Stolz, "Wigner theorems for random matrices with dependent entries: ensembles associated to symmetric spaces and sample covariance matrices," Electronic Communications in Probability, vol. 13, pp. 401-414, 2008.

[9] O. Friesen and M. Löwe, “The semicircle law for matrices with dependent entries".
[10] A. Zee, Quantum Field Theory in a Nutshell, Princeton University Press, Princeton, NJ, USA, 2nd edition, 2010.

[11] E. Brezin, C. Itzykson, G. Parisi, and J. B. Zuber, "Planar diagrams," Communications in Mathematical Physics, vol. 59, no. 1, pp. 35-51, 1978.

[12] E. Kanzieper, "Replica Approach in Random Matrix Theory," in The Oxford Handbook of Random Matrix Theory, Oxford Univ. Press, Oxford, UK, 2011.

[13] A. Zee, "Law of addition in random matrix theory," Nuclear Physics B, vol. 474, no. 3, pp. 726-744, 1996.

[14] J. Zinn-Justin, "Quantum field theory and critical phenomena," in International Series of Monographs on Physics, vol. 113, Oxford University Press, 2002. 

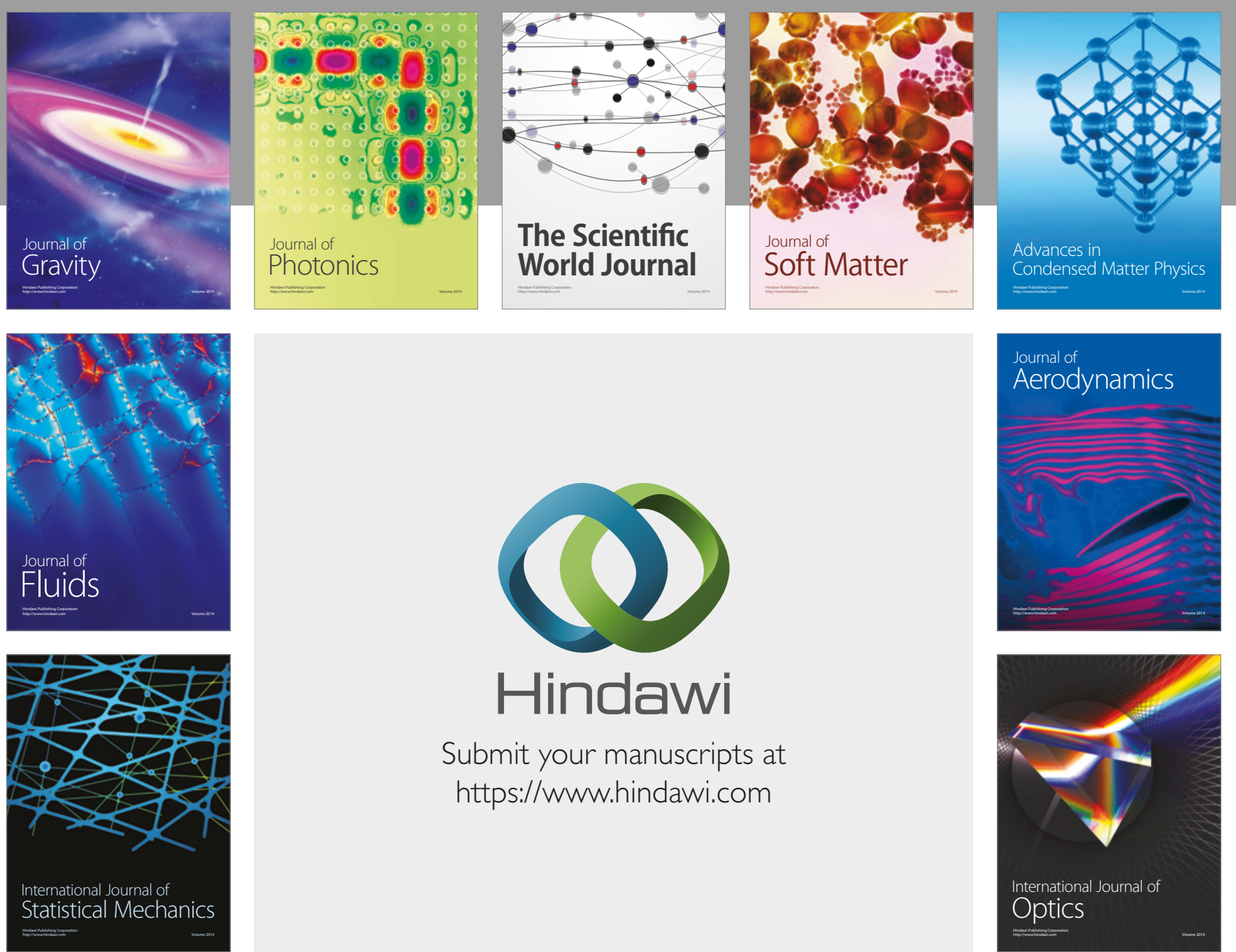

Submit your manuscripts at

https://www.hindawi.com
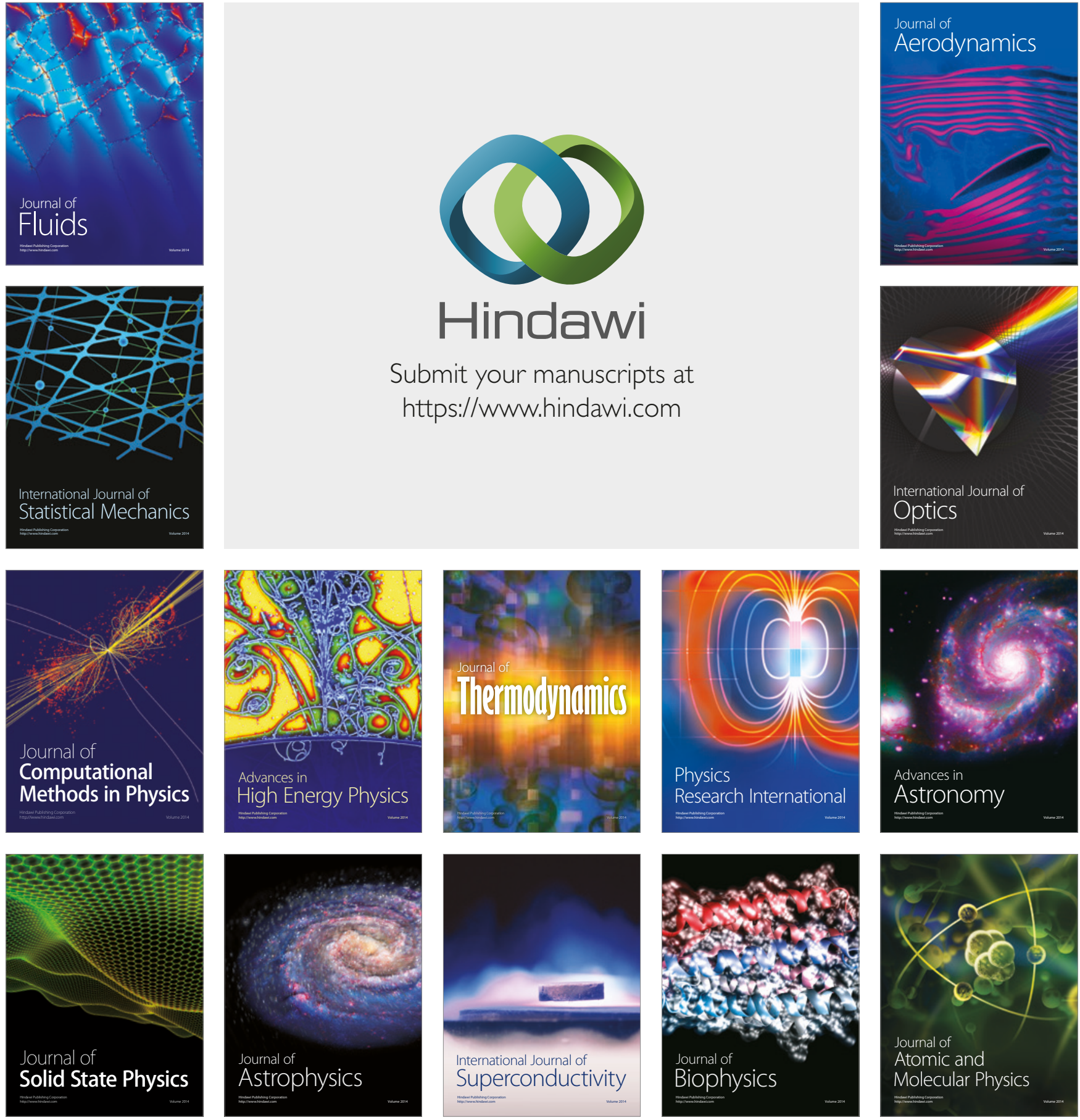